\title{
APPLICATION OF COMMUNICATION SYSTEMS IN BIOMEDICAL ENGINEERING
}

Picture archiving and communication systems (PACS) in biomedical engineering consist of image and data acquisition, storage, and display subsystems integrated by various digital networks. The standard (DICOM 3) is applicable to a networked or an off-line media environment. It allows development and expansion of PACS system. This Standard has been developed with an emphasis on diagnostic medical imaging as practiced in radiology, cardiology and related disciplines. Also the compression methods suitable for image processing are presented in this article. The compression is often realized by reduction of irrelevance or redundancy. There are described lossless and lossy compression methods which can be used for compression of images in biomedical applications and comparison of these methods based on fidelity criteria.

\section{Introduction}

Communication is the transport of information between two places, usually by means of some type of medium. A communication standard encompasses detailed specifications of the media, the explicit physical connections, the signal levels and timing, the packaging of the signals, and the high level software required for the transport. A video communication standard describes the characteristics of composite video signals including interlace or progressive scan, frame rate, line and frame retrace times, number of lines per frame, and number of frames per second. In a communication system PACS, the soft copy display is source of video signals; depending on the types of used monitor. These video signals will follow certain standards. At present DICOM 3.0. is standard in a PACS system.

\section{Picture Archiving and Communication Systems (PACS)}

A picture archiving and communication system (PACS) consists of image and data acquisition, storage, and display subsystems integrated by various digital networks (Fig.1) [1]. Each of PACS modules functioned as an independent island, unable to communicate with other modules. A general multimedia data management system that is easily expandable, flexible, and versatile in its programmability calls for both top-down management to integrate various information systems.

The PACS infrastructure provides the necessary framework for the integration of distributed and heterogeneous imaging systems and makes possible intelligent database management of all related information. The PACS infrastructure consists of a basic skeleton of hardware components (acquisition interfaces, storage devices, host computers, communication networks, and display systems) integrated by standardized, flexible software subsystems for com- munication, database and storage management, job scheduling, interprocessor communication, and network monitoring.

The PACS infrastructure is physically composed of several classes of computer systems connected by various networks. These include imaging systems, acquisition computers, and the PACS controller with database and archive, and display workstations.

The most troublesome PACS task to date has been the reliable and timely acquisition from an imaging system of images and associated study support text (description of the study, and parameters of acquisition and image processing).

This bottleneck exists mainly because many manufacturers of imaging equipment are not prepared to follow the industry standards developed by, for example, ACR-NEMA and DICOM. To circumvent these difficulties, an acquisition computer can be placed between the imaging system and the rest of the PACS network. The acquisition computer has three primary tasks: it acquires image data from the imaging system, converts the data from the equipment manufacturer's specifications to PACS standard format (header format, byte-ordering, matrix sizes) that is compliant with the proposed ACR-NEMA and DICOM data formats, and forwards the image study to the PACS controller.

PACS display stations should fully use the resources and processing power of the entire PACS network. A station includes communication, database, display, resource management, and processing software.

There are four types of display station: high-resolution monitors, medium resolution stations for conferences, desktop stations, and high-resolution, hard copy print stations.

A basic function of any computer network is to provide an access path by which end users at one geographic location can

\footnotetext{
* L. Hargaš, M. Hrianka, P. Špánik

University of Žilina, Univerzitná 1, 010 26, Ž ilina, Slovakia, E-mail: Libor.Hargas@fel.utc.sk, Miroslav.Hrianka@fel.utc.sk, Pavol.Spanik@fel.utc.sk
} 


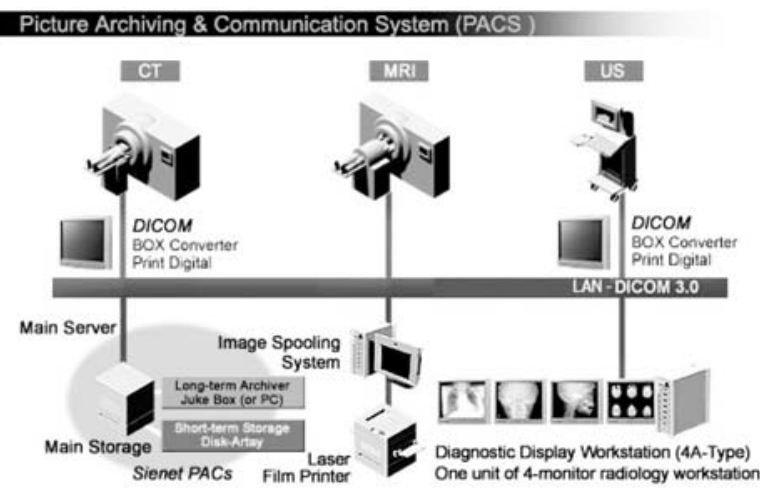

Fig. 1 PACS components

access information (e.g., images and reports) at another location. The most obvious way to characterize a PACS network is to examine the traffic of information between various locations and users. The important networking data needed for system design include location and function of each node, frequency of information passed between any two nodes, cost for transmission between nodes on various speed lines, desired reliability of the communication, and required throughput. The variables in the design include network topology, communication line capacities, and flow assignments.

\subsection{Image Acquisition}

Automated image acquisition from imaging devices to the PACS controller plays an important role in a PACS infrastructure. The „automatic" part is important because reliance on labor-intensive manual acquisition methods would defeat the purpose of the PACS. Based on existing manufacturers' imaging devices, we categorize the interface methods into five architectural models: sequential chain, direct interface, memory access, shared disk, and interconnected network.

DICOM Standard embodies a number of major enhancements to previous versions of the standard: is applicable to a networked or an off-line media environment; specifies how devices claiming conformance to the Standard react to commands and data being exchanged; is structured as a multi-part document, introduces explicit information objects not only for images and graphics but also for waveforms, reports, printing, etc.

\subsection{Goals of the DICOM standard}

The DICOM Standard facilitates interoperability of devices claiming conformance. In particular, it:

- Addresses the semantics of Commands and associated data. For devices to interact, there must be standards on how devices are expected to react to Commands and associated data, not just the information which is to be moved between devices;

- Addresses the semantics of file services, file formats and information directories necessary for off-line communication;
- Is explicit in defining the conformance requirements of implementations of the Standard. In particular, a conformance statement must specify enough information to determine the functions for which interoperability can be expected with another device claiming conformance.

- Facilitates operation in a networked environment.

- Is structured to accommodate the introduction of new services, thus facilitating support for future medical imaging applications.

- Makes use of existing international standards wherever applicable, and itself conforms to established documentation guidelines for international standards.

\section{Image compression in communication system}

Image compression involves reducing the size of image data files, while retaining necessary information. The ratio of the original, uncompressed image file and the compressed file is referred to as the compression ratio. The compression ratio is denoted by [2]

$$
\text { compresion ratio }=\frac{\text { size }_{U}}{\text { size }_{C}},
$$

where $s_{i z e}$ - uncompressed file size, size $_{C}$ - compressed file size.

Compression algorithms are developed by taking advantage of the redundancy that is inherent in image data. Three primary types of redundancy can be found in images: coding, inter pixel, psycho visual redundancy.

If we want to create a successful compression scheme, we must differentiate between data and information. For digital images, data refers to the pixel gray-level values that correspond to the brightness of a pixel at a point in space. Information is an interpretation of the data in a meaningful way. For example, in a binary image that contains text only, the necessary information may only involve the text being readable, whereas for a medical image the necessary information may be every minute detail in the original image.

The compression system model consists of two parts: the compressor and the decompressor. The compressor consists of a preprocessing stage and encoding stage, whereas the decompressor consists of a decoding stage followed by a postprocessing stage. Before encoding, preprocessing is performed to prepare the image for the encoding process, and consists of any number of operations that are application specific. After the compressed file has been decoded, postprocessing can be performed to eliminate some of the potentially undesirable artifacts brought about by the compression process.

\subsection{Basic compression technique}

Lossless compression methods

Lossless compression methods are necessary in some imaging applications. The most known algorithms are Huffman coding, Run-Length coding, LZW coding (Lempel-Ziv-Welch), arithmetic 


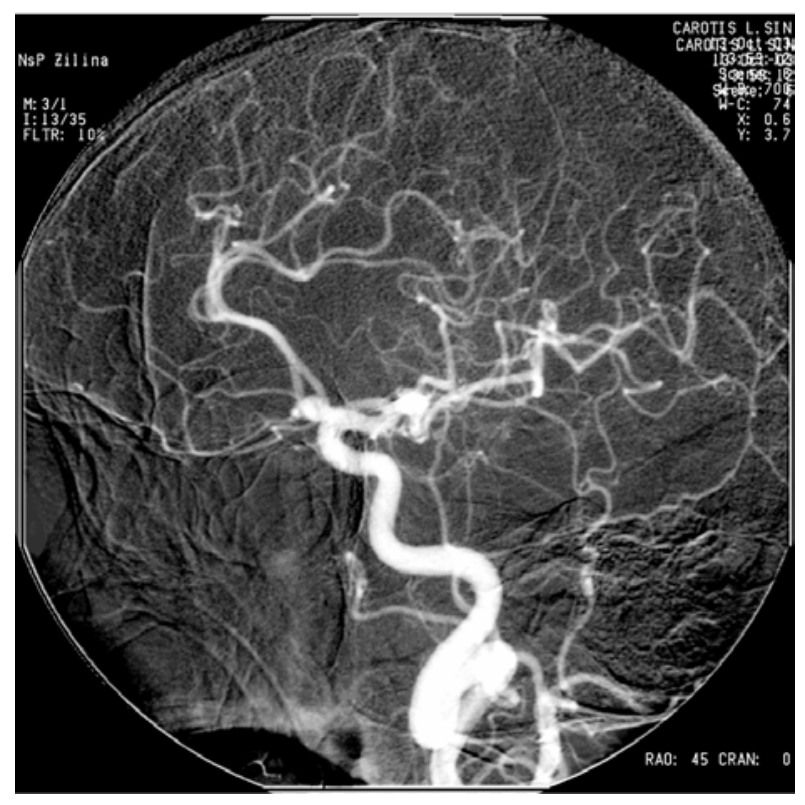

Fig. 2 Original image

coding [2]. In general, the lossless techniques alone provide marginal compression of complex image data, often in the range of only a $10 \%$ reduction in file size. Comparing compression methods are presented by original image Fig. 2 (Source: Hospital with Policlinic of Žlina). Many of the lossless techniques were developed for non-image data and, consequently, are not optimal for image compression.

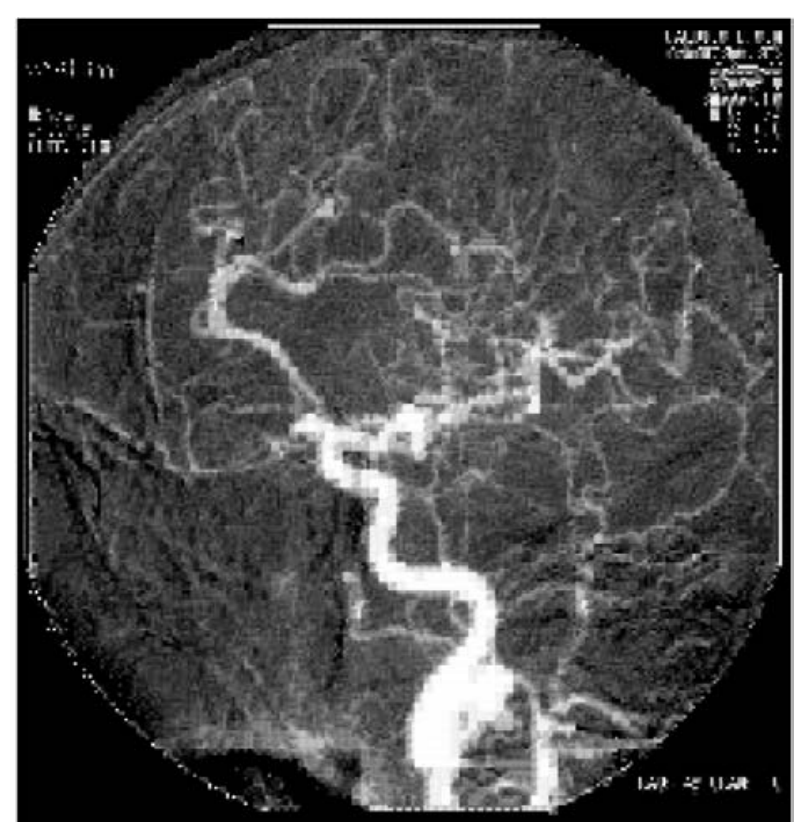

Fig. 4 VQ compression

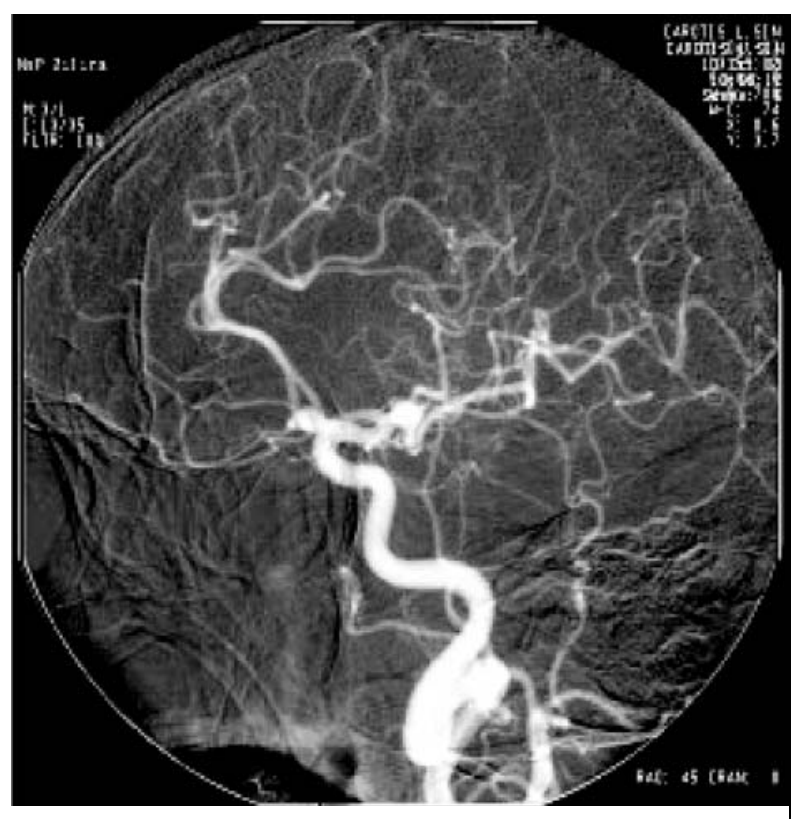

Fig. 3 Predictive BTC compression

Lossy compression methods

Lossy compression provides tradeoffs between image quality and degree of compression, which allows the compression algorithm to be customized to the application. With some of the more advanced methods, images can be compressed 10 to 20 times with virtually no visible information loss, and 30 to 50 times with minimal degradation. Many of the methods have adjustable parameters to allow the user to select the desired compression ratio and image fidelity [4]. Block truncation coding (BTC) works by dividing the image into small sub images and then reducing the number of gray levels within each block. This reduction is performed by a quantizer that adapts to the local image statistics. An example is presented in Fig. 3.

Vector quantization (VQ) is the process of mapping a vector that can have many values to a vector that has a smaller (quantized) number of values. For image compression, the vector corresponds to a small subimage, or block. Vector quantization treats the entire subimage (vector) as a single entity and quantizes it by reducing the total number of bits required to represent the subimage. An example with $3 \times 3$ subimage is presented in Fig. 4.

\subsection{Fidelity criteria}

Fidelity criteria can be divided into two classes: objective fidelity criteria, subjective fidelity criteria. The objective fidelity criteria are borrowed from digital signal processing and information theory and provide us with equations that can be used to measure the amount of error in the reconstructed image. Subjective fidelity criteria require the definition of a qualitative scale to assess image quality. This scale can then be used by human test subjects to determine 
image fidelity. The objective criteria, although widely used, are not necessarily correlated with our perception of image quality [1].

Commonly used objective measures are the root-mean-square error $e_{R M S}$, the root-mean-square signal-to-noise ratio $S N R_{R M S}$, the peak signal-to-noise ratio $S N R_{P E A K}$.

$$
\begin{aligned}
& e_{R M S}=\sqrt{\frac{1}{N^{2}} \sum_{r=0}^{N-1} \sum_{c=0}^{N-1}[I(r, c)-I(r, c)]^{2}} \\
& S N R_{R M S}=\sqrt{\frac{\sum_{r=0}^{N-1} \sum_{c=0}^{N-1}[I(r, c)]^{2}}{\sum_{r=0}^{N-1} \sum_{c=0}^{N-1}[I(r, c)-I(r, c)]^{2}}} \\
& S N R_{P E A K}=10 \log _{10}=\frac{1}{\frac{1}{N^{2}} \sum_{r=0}^{N-1} \sum_{c=0}^{N-1}[I(r, c)-I(r, c)]^{2}}
\end{aligned}
$$

where $I(r, c)$ is the original image, $I(r, c)$ is the decompressed image and $N \times N$ image size.

These objective measures are often used in research because they are easy to generate and seemingly unbiased, but remember that these metrics are not necessarily correlated to our perception of an image.

Subjective testing is performed by creating a database of images to be tested, gathering a group of people that are representatives of the desired population. Subjective fidelity measures can be classified into three categories: impairment, quality and comparison tests [2].

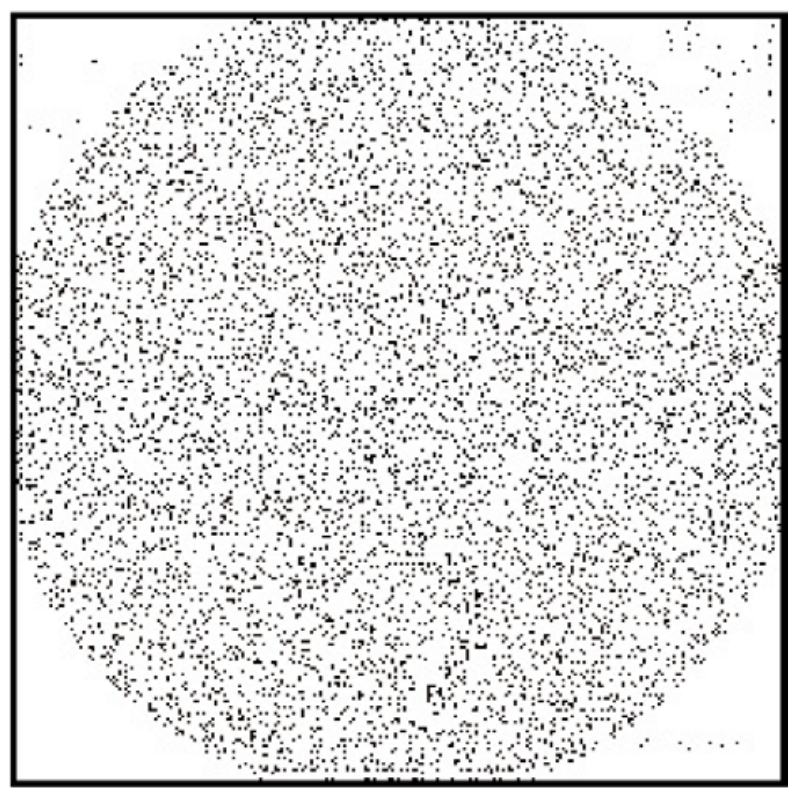

Fig. 5 Difference image Fig. 2 and Fig. 3
The comparison is done for block truncation coding (BTC) and vector quantization (VQ). In Fig. 5 and Fig. 6 are illustrated lost data, caused by compressions. These images were obtained by comparison of an original image with a compressed image, using function subtraction (contrast is increased for print matter).

\section{Conclusion}

A PACS module is loosely defined as a self-contained PACS that has some acquisition components: a short-term archive, a database, some display stations, and a communication network linking these components. In practice, the module can function alone, as an individual unit in which the display stations show images from the acquisition components.

There are two categories of preprocessing function. The first is related to the image format - for example, a conversion from the manufacturer's format to DICOM. The second type of preprocessing prepares the image for an optimal viewing at the display station.

Some approaches of image compression methods by the fidelity criteria are presented in these images. These criteria, although widely used, are not necessarily correlated with subjective perception of image quality. Subjective fidelity criteria require the definition of a qualitative scale to assess image quality. This scale can then be used by human test subjects to determine image fidelity.

\section{Acknowledgement}

This work was supported by the grant No. 1/3107/06 from the VEGA grant agency.

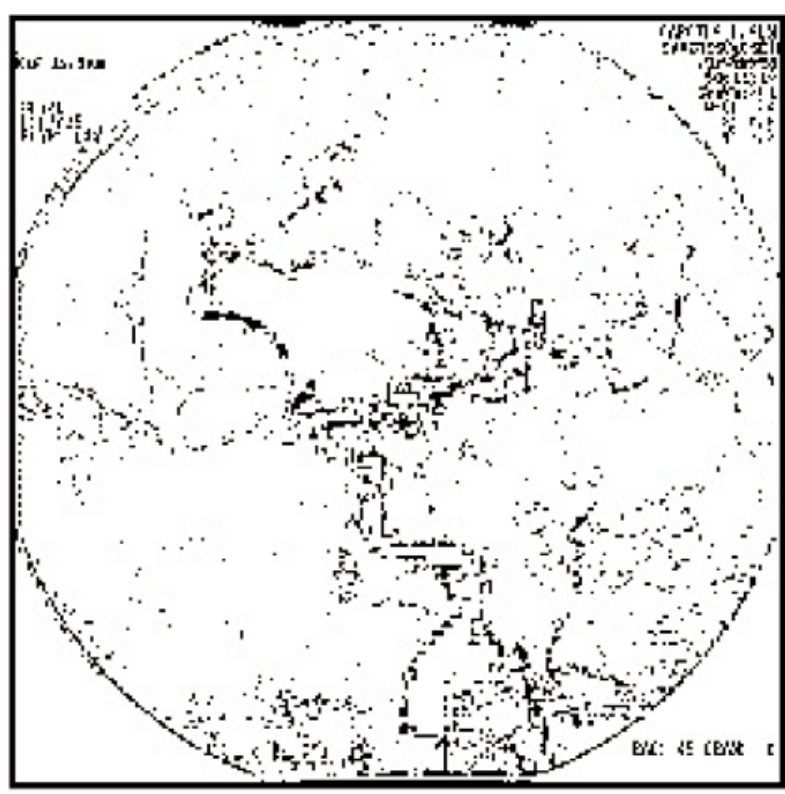

Fig. 6 Difference image Fig. 2 and Fig. 4 


\section{References:}

[1] HUANG, H. K.: Picture Archiving and Communication Systems in Biomedical Imaging, VCH Publishers, Inc., 1996

[2] UMBAUGH, S. E.: Computer Vision and Image Processing, Prentice Hall PTR, 1999

[3] CVIP: www.ee.siue.edu/CVIPtools

[4] NEMA National Electrical Manufacturers Association, www.nema.com, Digital Imaging and Communications in Medicine, Rosslyn, Virginia, 2003

[5] CASTELMAN, K. R.: Digital Image Processing, Prentice Hall, 1996

[6] RUSS, J. C.: The Image Processing, CRC Press LLC, 1999 